\title{
The Impact of Stock Liquidity on Firm Innovation: Evidence From China
}

\author{
Ni Zhong ${ }^{1}$ \\ ${ }^{1}$ School of Economics, Jinan University, Guangzhou, China \\ Correspondence: Ni Zhong, School of Economics, Jinan University, Guangzhou, China. Tel: 86-137-1039-0414.
}

Received: March 13, 2017

Accepted: March 28, 2018

Online Published: April 9, 2018

doi:10.20849/ajsss.v3i2.357

URL: https://doi.org/10.20849/ajsss.v3i2.357

\begin{abstract}
Stock market has become a crucial financing channel for firms nowadays. As an important dimension of the characteristics of stock market, how will stock liquidity affect firm innovation? What are the underlying mechanisms? Those questions need to be explored further. Using Chinese non-financial A-share listed companies which had disclosed R\&D expenditures in year 2006 to 2016 as the study sample, this paper investigates the effect of stock liquidity on firm innovation. The result shows that stock liquidity has a significant and positive effect on firm innovation. My finding is supported by additional test using Heckman sample selection model and several robustness tests. Further analysis shows that stock liquidity improves firm innovation by reducing financing constraints and increasing agency costs. This paper deepens the research on firm innovation from the perspective of market microstructure and owns implication for the government to encourage investment in R\&D and will lead to develop multi-tiered capital markets.
\end{abstract}

Keywords: stock liquidity, firm innovation, financing constraints, agency costs, mediating effects

JEL Classification: G19, G34, G38, O32

\section{Introduction}

The importance of innovation for a country has been widely recognized. Advancement of science and technology is critical for economy updating and sustainable economic growth. For China, the largest developing country in the word with many economic growth problems, innovation is particularly important. With economic growth slowing to a "new normal", in order to adapt to the new changes, changing the mode of economic development, science and technology are the keys. Firms are the main force of innovation. Thus, it is urgent to figure out the factors that determine and influence firm innovation.

At present, the factors that influence firm innovation have been studied extensively. Lu and Dang (2004) find that corporate governance significantly affects firm's R \& D investment. Chen, Li, and Bai (2007) find that market competition significantly affects R\&D input. Liu, Lin, Shun, and Chen (2015) by using the two-tier stochastic frontier model, find that financing constraints are negatively correlated with R\&D investment, and agency costs are positively correlated with R\&D investment. Xiao (2016) finds that the ownership structure and the size of the board of directors significantly affect $R \& D$ investment.

Most of the existing researches' results are focused on macro factors, industry factors and firm internal factors. In recent years, the academia has begun to realize the importance of stock market to $\mathrm{R} \& \mathrm{D}$ investment and began to study firm innovation from the perspective of market microstructure. Stock liquidity, as one of the most important characteristics of stock market, is thought to be one of the factors that may affect the innovation activities of firms. But when analysing stock liquidity and firm innovation, most of the researchers use the innovation output as the measure of firm innovation, while some use innovation input. Using patenting activity as a measure of firm innovation, Fang, Tian, and Tice (2014) argue a negative correlation between stock liquidity and firm innovation, Dass, Nanda, Vikram, and Xiao (2012), Lai (2014), Feng, Liu, Feng, and Wen (2017) argue a positive correlation between stock liquidity and firm innovation. There is no consensus about whether the relationship between stock liquidity and firm innovation is negative or positive. In addition, there are few literatures studying the underlying mechanism in China. Further research is needed. In this context, this paper analyses the relationship between stock liquidity and firm innovation with panel data of the annual reports of Chinese A-share listed firms from 2006 to 2016 and further analyses its mechanisms. 
My study has the following contributions: (i) this paper provides one more new empirical evidence on the economic consequences of stock liquidity; (ii) in order to make my study more in line with the characteristics of Chinese financial market (Investors in Chinese financial market are more likely to pursue short-term interests) and the Chinese firm's conditions (Chinese firms are facing severe external financing constraints and internal governance deficiencies), I examine the possible underlying mechanisms: financing constraints mechanism and agency costs mechanism. Thus, the study's findings should be more robust and valid.

The remaining part of this paper is organized as follows. In Section 2, after theoretical analysis and logical reasoning, I make my hypotheses; Section 3 presents research design and variable description; Section 4 reports basic empirical result; Section 5 provides additional evidence and makes robustness tests; Section 6 makes further mechanism tests; and Section 7 summarizes the conclusions and policy implications.

\section{Motivation and Testable Hypotheses}

The liquidity of the stock is the ability of the market to carry out the stock exchanges at a reasonable price. Recent studies have shown that stock liquidity is closely related to the internal factors of a firm. Cheung, Chung, and Fung (2015) find that stock liquidity affects corporate governance by affecting the proportion of institutional investors. Xiong and Su (2014) find that the stock liquidity alleviates firms' underinvestment and restrain overinvestment. Xiong and Su (2016) find that stock liquidity can reduce the agency costs by using the stochastic frontier model. As one kind of firm investment, innovation investment not only has general characteristics as other kinds of investment, but also has its own characteristics. Like other investments, innovation investment is also affected by financing constraints, agency costs and other factors in the firms' level. At the same time, since its high risk, strong asset specificity and long cycle characteristics, innovation investment has more uncertainty. Schumpeter's innovation theory suggests that credit system is the economic condition for entrepreneurs to achieve innovation, and the establishment of capital market is the foundation for innovation. As part of the capital market, the stock market is bound to affect the innovation activities of a firm. As an important characteristic of stock market, the stock liquidity may be one of the important factors that affects the innovation activities of a firm.

According to the existing researches and theories, I find that the stock liquidity may affect the firm's innovation through the following ways.

First of all, stock liquidity may affect the firm's innovation through the financing constraint mechanism. Innovation activities require huge initial capital investment. Additionally, subsequent formation of new ideas, new product concept, and new product development all require more investment. Yet, the supply of internal fund is limited and unstable, there is a need for external financing to access sufficient funds. On one hand, there are many uncertainties in the process of innovation activities, and the risk of failure is high. On the other hand, since innovation can be easily imitated, the innovation activities of firms are confidential, and firms are not willing to disclose their innovation ideas. Thus, the investment process is highly asymmetric. External investors are not fully aware of the possibility of the success of the innovation investment. Since the complexity of research and development, even if some details are disclosed, external investors may not be able to make full use of the information disclosed to make a trade-off between risk and return. In addition, the high level of asset specificity and huge funds inputting in human capital and intangible assets lead to the lack of financing guarantees. These factors lead to higher financing cost of firm innovation activities, and high degree of external financing constraints, which is quite unfavourable to firm innovation. Zhang, Lu, Zheng, and Chen (2012), Lu, Zheng, and Li (2013), Liu, Lin, Shun, and Chen (2015), Chen and Wang (2016) all find that financing constraints have a significant negative effect on $R \& D$ investment of firms.

The liquidity premium theory which was put forward by Amihud and Mendelson (1986), suggests that high liquidity can reduce capital cost. In addition, the reduced information asymmetry resulted by high stock liquidity will also ease the financing constraints. Those analyses above suggest that high stock liquidity may positively affect firm innovation through the financing constraints mechanism.

Secondly, stock liquidity may affect the firm's innovation through the agency costs mechanism. The separation of ownership and management leads to the principal-agent problem. Executives tend to build personal empire through overinvestment. The agency problem exists in the innovation investment too. On one hand, for their own interests, executives tend to reduce investment in projects with long term and high risk, such as innovation projects. Lv (2014), Luo, Li, and Chang (2014) find that agency costs affect firm innovation negatively. On the other hand, the motivation of building "Enterprise Empire" and controlling more resources to gain more personal interests will also impel executives to overinvest. In addition, innovation investment can act as a means to profit budgeting (Liu, 2007). Liu et al (2015) find there is a significant positive correlation between agency costs and R\&D investment. Those suggest that the agency problem can affect firm innovation. 
Stock liquidity can affect agency costs. On one hand, when stock liquidity is high, executives tend to reduce opportunism behaviours in order to reduce the adverse impact by the change of liquidity on their income. At the same time, the increase of liquidity reduces information asymmetry and strengthens the supervision of the executives by external investors. But on the other hand, the higher liquidity means the lower transaction cost of the selling stocks. Large shareholders and institutional investors are more likely to exit the company (Bhide, 2004). Stock markets tend to pursue short-term interests. This feature is more significant in the Chinese stock market. When the stock liquidity is high, shareholders are more likely to pursue short-term return rather than supervising the behaviours of the executives. On the contrary, when the stock liquidity is low, there is an exit barrier which may lead to "forced supervision". Those analyses suggest that high stock liquidity may affect firm innovation through the agency costs mechanism, while it cannot be sure that whether the effect is negative or not.

While the above analyses suggest that stock liquidity could have ambiguous effect on firm innovation, I make predictions based on the overall weight of the empirical evidence. My hypotheses are as follows:

H1: Firm with higher stock liquidity have a higher level of innovation.

H2: Firm with higher stock liquidity have a lower level of innovation.

\section{Research Design}

\subsection{Innovation Measures (LNRD/RDIN)}

There are two kinds of methods to measure a firm's innovation: R\&D expenditures and patenting activity. These two measures have their own merits and demerits. R\&D expenditures only measure the input aspect of a firm's innovation, but it is more directly related to the firm's characteristics. Patenting activity can measure the innovation output and efficiency but it is affected by far more uncertain factors than R\&D expenditures. In this paper, I will analyse the impact of management behaviour on the firm innovation. Compared with innovation input, innovation output is less directly controlled by the firm management. Therefore, for the purposes of my paper, I choose R\&D expenditures to measure innovation. Here I adopt three kinds of methods to measure R\&D expenditures: LNRD which is the natural logarithm of R\&D investment, RDIN which is the ratio of R\&D investment and revenue, and RDTA which is the ratio of R\&D investment and total assets. Among them, RDTA is a variable for robustness test.

\subsection{Liquidity Measures (LIQ)}

Considering the availability and cost of data and comparing the merits and demerits of many stock liquidity measures, I use the following 5 methods to measure stock liquidity:

\section{(1) Proportions of Zero Return Days}

Proportions of Zero Returns Days (denoted as ZERO) is a measure of stock illiquidity developed by Lesmond, Ogden, and Trzcinka (1999). The formula is:

$$
Z E R O_{i t}=\frac{N_{i t}}{D_{i t}}
$$

where $N_{i t}$ is the number of days with zero returns for stock i in a year, $D_{i t}$ is the number of trading days for stock $\mathrm{i}$ in year $\mathrm{t}$.

\section{(2) Liu's Indicator}

Liu (2005) defined a new liquidity measure (denoted as LIU) of a security. It measures many dimensions of liquidity and emphasizes the transaction speed which is ignored by many liquidity indicators. The formula is:

$$
\begin{aligned}
& \text { LIUX }_{i}=\left[\text { ZeroTD }_{i}+\frac{1}{x-\text { month Turnover }_{i}} \cdot \frac{1}{\text { Deflator }^{2}}\right] \times \frac{21 x}{\text { NoTD }_{i}} \\
& x-\text { month }_{\text {Turnover }}=\sum \frac{\text { Vol }_{i t}}{\text { Moveshare }_{i t}}
\end{aligned}
$$

where $\operatorname{ZeroTD}_{i}$ is the number of days with zero daily volumes in prior $\mathrm{x}$ months, $x$ - month Turnover $_{i}$ is the turnover over the prior $\mathrm{x}$ month, which is calculated as the sum of daily turnover over the prior $\mathrm{x}$ months. NoTD is the number of trading days in the market over the prior x months. Deflator acts as an adjustment which is chosen such that

for all sample stocks. In this paper, I use a deflator of 11,000.

$$
0<\frac{1}{x-\text { month Turnover }_{i}} \cdot \frac{1}{\text { Deflator }_{\text {The }}}<1
$$




\section{(3) Return Reversal Indicator}

Return Reversal Indicator (denoted as GAM) is developed by Pastor and Stambaug (2003). The indicator can be estimated by the equation below.

$$
r_{i, t, d+1}^{e}=\theta_{i, t}+\phi_{i, t} r_{i, t, d}+\gamma_{i, t} \cdot \operatorname{sign}\left(r_{i, t, d}^{e}\right) \cdot v_{i, t, d}+\varepsilon_{i, t, d+1}
$$

where $r_{i, t, d}$ is the return on stock i on day d in month t. $r_{i, t, d}^{e}$ is the excess return, which is the difference between stock return and market return: $r_{i, t, d}^{e}=r_{i, t, d}-r_{m, t, d}, r_{m, t, d}$ is the return on the Chinese-A-share value-weighted market return on day $\mathrm{d}$ in month t. $v_{i, t, d}$ is the trading volume for stock $\mathrm{i}$ on day $\mathrm{d}$ in month $\mathrm{t}$. GAM is the absolute value of $\gamma_{i, t}$. For a given trading volume, the lower the stock liquidity, the greater the possibility of return reversal.

(4) Amihud Illiquidity Ratio

Amihud illiquidity ratio (denoted as AMIHUD) is developed by Amihud. The formula is:

$$
A M I H U D_{i t}=\frac{1}{D_{i t}} \sum_{d=1}^{D_{i t}}\left(\frac{\left|r_{i t d}\right|}{V_{i t d}}\right) \times 100
$$

where $D_{i t}$ is the number of trading days for stock i in year $\mathrm{t}, r_{i t d}$ is the return on stock i on day d in year $\mathrm{t}, V_{i t d}$ is the trading volume for stock i on day d of year t. $\left|r_{i t d}\right| / V_{i t d}$ is the ratio which gives the price change per unit of daily trading volume, or the daily price impact of the order flow.

\section{(5) Amihud Square Root Indicator}

To reduce the effect of the extreme numerical values when using the Amihud illiquidity ratio, in this paper, I also use the Amihud square root indicator (denoted as AMIHUDSR) to measure stock illiquidity. The formula is:

$$
A M I H U D S R_{i t}=\frac{1}{D_{i t}} \sum_{d=1}^{D_{i t}} \sqrt{\left(\frac{\left|r_{i t d}\right|}{V_{i t d}} \times 10^{6}\right)}
$$

where $D_{i t}$ is the number of trading days for stock i in year $\mathrm{t}, r_{i t d}$ is the return on stock i on day d in year $\mathrm{t}, V_{i t d}$ is the trading volume for stock $\mathrm{i}$ on day $\mathrm{d}$ of year $\mathrm{t}$.

\subsection{Other Variables}

I choose the widely used ratio of administrative expenses to total asset as the proxy for the agency problem and the asset-liability ratio as the proxy for financial constraints.

In order to control for those firm and industry characteristics that may affect a firm's innovation, following the current literatures on innovation, I include several control variables: firm's age, capital expenditure ratio, size, return on equity ratio, Tobin's Q, fixed assets, executives share, executive compensation, market competition, employee, Lerner index, intangible assets, cash flow, cash stock, liquidity ratio, ownership concentration indicator. In detail, firm's age is the natural logarithm of age. Capital expenditure ratio is the ratio of capital expenditures to total assets. Size is the natural logarithm of total assets. Return on equity ratio is the ratio of net profits to equity. I calculate the Tobin's $Q$ as the ratio of market value of equity to total assets. Fixed asset is the ratio of net property, plant and equipment (PPE) to total assets. Executives share is calculated as shares holding by executives divided by total share. Executive compensation is calculated as the natural logarithm of the sum of the top three executives' compensations. Market competition is calculated as selling expenses divided by revenue. Employee is the number of employees. Lerner index is calculated as: (operating income - operating costs the cost of sales management fees) / operating income, to measure the market structure. Intangible assets is the ratio of intangible assets to total assets. Cash flow is the ratio of operating-activity cash flow to fixed asset. Cash stock is the ratio of cash to fixed assets. Liquidity ratio is current assets divided by current liability. Ownership concentration indicator is the sum of the squares of the top 5 executives' shareholding proportion. In addition, industry and yearly fixed effects are controlled in the model.

\subsection{Model Design}

In order to analyse the relationship between stock liquidity and firm R\&D investment, I construct the following regression model:

$$
L N R D_{i t}\left(R D I N_{i t}\right)=\varphi_{0}+\varphi_{1} L I Q_{i t}+\varphi \operatorname{Control}_{i t}+\varepsilon_{i t}
$$

where $L N R D_{i t}$ is the natural logarithm of $\mathrm{R} \& \mathrm{D}$ expenditures, $R D I N_{i t}$ is the ratio of $\mathrm{R} \& \mathrm{D}$ expenditures to revenue. $L I Q_{i t}$ is the stock liquidity of stock i in year t. Control $_{i t}$ is control variable. 


\subsection{Data and Descriptive Statistics}

Before 2006, only few firms disclosed R\&D expenditures in China. Therefore, I set my study period from 2006 to 2016. Sample selection is based on the following criteria: (i) firms in the financial sectors are excluded; (ii) firms with incomplete data are excluded. All data are obtained from the database and sub database of CSMAR and Wind about the Chinese-A-share listed firms. In order to mitigate the effect of extreme values, I use Stata13.1 to winsorize major variables by $1 \%$ and $99 \%$ before regression. Table 1 shows the descriptive statistics and the definitions of each variable.

\section{Empirical Result}

I first apply the OLS method in my regression. Table 2 reports the regression results.

In Panel A, the dependent variable is LNRD. Independent variables are Proportions of Zero Return Days, LIU's Indicator, Return Reversal Indicator, Amihud Illiquidity Ratio, Amihud Square Root Indicator in columns (1) to columns (5) respectively. The coefficients on all five illiquidity measures are negative and both economically and statistically significant. Among them, the coefficients on LIU, GAM, AMIHUD and AMIHUDSR are significant at the $1 \%$ level. Panel B of table 2 reports the regression results with the dependent variable replaced by RDIN. The coefficients on four illiquidity measures are negative and both economically and statistically significant. This suggests that firms with greater stock liquidity have a higher innovation level. H1 is supported.

To control the influence of the unobservable factors at the firm level, I also apply the fixed effect regression method. Table 3 reports the regression results. In Panel A, the dependent variable is LNRD. The coefficients on LIU, GAM and AMIHUD are negative and both economically and statistically significant. Panel B of table 3 reports the regression results with the dependent variable replaced by RDIN. The coefficients on AMIHUD and AMIHUDSR are negative and both economically and statistically significant. This suggests that even after controlling those unobservable factors the conclusion that firm with greater stock liquidity have a higher innovation level still valid.

Table 1. Variable definition and descriptive statistics (2006-2016)

\begin{tabular}{lllllll}
\hline Variable & Definition & Obs. & Mean & Std. Dev. Min. & Max. \\
\hline LNRD & Natural logarithm of R\&D expenditures15017 & 17.21 & 1.605 & 12.68 & 21.13 \\
RDIN & R\&D expenditures/revenue & 14926 & 0.0365 & 0.0391 & 0.000162 & 0.225 \\
RDTA & R\&D expenditures/total assets & 14926 & 0.0186 & 0.0178 & $8.48 \mathrm{e}-05$ & 0.122 \\
ZERO & Proportions of Zero Return Days & 15028 & 0.0203 & 0.0187 & 0 & 0.109 \\
LIU & LIU's Indicator & 14926 & 22.2078 & 51.5791 & $5.47 \mathrm{e}-08$ & 306.98 \\
GAM & Return Reversal Indicator & 14993 & 0.0783 & 0.230 & 0.000338 & 2.372 \\
AMIHUD & Amihud Illiquidity Ratio & 15008 & 0.174 & 0.663 & 0.00273 & 5.147 \\
AMIHUDSR & Amihud Square Root Indicator & 15028 & 0.0217 & 0.0169 & 0.00465 & 0.133 \\
LEV & Asset-liability ratio & 14926 & 0.409 & 0.213 & 0.0516 & 1.112 \\
MCOST & Administrative expenses/total asset & 15005 & 0.104 & 0.0869 & 0.00870 & 1.055 \\
LNAGE & Natural logarithm of age. & 14926 & 2.698 & 0.351 & -1.199 & 4.190 \\
CAPEXTA & Capital expenditures/total assets & 14923 & 0.0572 & 0.0501 & 0.000104 & 0.252 \\
SIZE & Natural logarithm of total assets. & 14926 & 21.84 & 1.219 & 18.21 & 25.49 \\
ROE & Net profits/equity & 14867 & 0.0756 & 0.118 & -0.578 & 0.624 \\
Q & Tobin's Q & 14585 & 2.535 & 2.157 & 0.166 & 12.71 \\
PPETA & PPE/total assets & 14925 & 0.224 & 0.151 & 0.00255 & 0.732 \\
MSHARE & Executives shares/total share & 14493 & 0.0812 & 0.149 & 0 & 0.595 \\
MPAY & Natural logarithm of executive payment14989 & 14.08 & 0.698 & 11.73 & 16.11 \\
COMPETITIONSelling expenses/revenue & 14983 & 0.0720 & 0.0787 & 0 & 0.429 \\
EMPLOYEE & The number of employees & 14926 & 4591 & 8102 & 36 & 49890 \\
PCM & Lerner index & 14983 & 0.0998 & 0.130 & -0.953 & 0.531 \\
INTANGTA & Intangible assets/total assets & 14794 & 0.0468 & 0.0447 & $4.79 \mathrm{e}-05$ & 0.319 \\
CF & CFO/fixed assets & 14925 & 0.291 & 1.746 & -13.42 & 17.77 \\
CFSTOCK & Cash/fixed assets & 14925 & 3.005 & 7.897 & 0.0236 & 72.03 \\
LDR & Current asset/ current liability & 14926 & 2.807 & 3.062 & 0.217 & 16.43 \\
SHRHFA5 & Ownership concentration indicator & 15028 & 0.168 & 0.115 & 0.0132 & 0.566 \\
\hline
\end{tabular}


Table 2. Stock liquidity and firm innovation (OLS specifications)

Panel A. Innovation measured by LNRD

\begin{tabular}{|c|c|c|c|c|c|}
\hline & $\begin{array}{l}(1) \\
\text { 7FRO }\end{array}$ & (2) & (3) & $\begin{array}{l}\text { (4) } \\
\text { MIHUD }\end{array}$ & $\begin{array}{l}\text { (5) } \\
\text { AMUHDSR }\end{array}$ \\
\hline \multirow{2}{*}{ LIQ } & $-1.403^{* *}$ & $-0.00113^{* * * *}$ & $-0.110^{* * * *}$ & $-0.0545^{* * * *}$ & $-2.282^{* * * *}$ \\
\hline & $(-1.98)$ & $(-4.87)$ & $(-3.51)$ & $(-4.92)$ & $(-4.38)$ \\
\hline \multirow[t]{2}{*}{ LNAGE } & $-0.321^{* * *}$ & $-0.327^{* * *}$ & $-0.331^{* * *}$ & $-0.333^{* * *}$ & $-0.329^{* * *}$ \\
\hline & $(-11.01)$ & $(-11.22)$ & $(-11.27)$ & $(-11.38)$ & $(-11.27)$ \\
\hline \multirow{2}{*}{ CAPEXTA } & $1.387^{* * * *}$ & $1.399^{* * * *}$ & $1.423^{* * * *}$ & $1.427^{* * *}$ & $1.427^{* * * *}$ \\
\hline & $(6.05)$ & $(6.15)$ & $(6.24)$ & $(6.27)$ & $(6.27)$ \\
\hline \multirow{2}{*}{ SIZE } & $0.568^{* * * *}$ & $0.565^{* * * *}$ & $0.559^{* * *}$ & $0.559^{* * *}$ & $0.548^{* * *}$ \\
\hline & $(34.01)$ & $(34.17)$ & (33.34) & (33.48) & (31.94) \\
\hline \multirow[t]{2}{*}{ ROE } & $1.267^{* * * *}$ & $1.264^{* * * * * *}$ & $1.290^{* * * * *}$ & $1.298^{* * * *}$ & $1.293^{* * * * *}$ \\
\hline & $(9.00)$ & $(8.97)$ & (9.18) & $(9.22)$ & $(9.20)$ \\
\hline \multirow[t]{2}{*}{ Q } & 0.00384 & 0.00796 & 0.00525 & 0.00834 & 0.00479 \\
\hline & $(0.61)$ & $(1.25)$ & $(0.82)$ & $(1.28)$ & $(0.76)$ \\
\hline \multirow[t]{2}{*}{ PPETA } & $-0.908^{* * * *}$ & $-0.940^{* * *}$ & $-0.936^{* * *}$ & $-0.930^{* * *}$ & $-0.925^{* * *}$ \\
\hline & $(-9.63)$ & $(-10.16)$ & $(-10.10)$ & $(-10.04)$ & $(-9.99)$ \\
\hline \multirow[t]{2}{*}{ MSHARE } & $0.481^{\text {****** }}$ & $0.486^{* * *}$ & $0.504^{* * * * *}$ & $0.507^{* * * *}$ & $0.509^{* * * * *}$ \\
\hline & $(8.90)$ & $(9.01)$ & $(9.29)$ & $(9.38)$ & $(9.43)$ \\
\hline \multirow[t]{2}{*}{ MPAY } & $0.391^{* * * *}$ & $0.393^{* * * *}$ & $0.395^{* * * *}$ & $0.394^{* * * *}$ & $0.393^{* * * *}$ \\
\hline & (22.23) & (22.38) & (22.44) & $(22.40)$ & (22.39) \\
\hline \multirow[t]{2}{*}{ COMPETITION } & 0.0614 & 0.0443 & 0.0543 & 0.0314 & 0.0374 \\
\hline & $(0.50)$ & $(0.36)$ & $(0.44)$ & $(0.25)$ & $(0.30)$ \\
\hline \multirow[t]{2}{*}{ EMPLOYEE } & $2.25 \mathrm{e}-05^{5^{* *}}$ & $2.19 \mathrm{e}-05^{* * *}$ & $2.25 \mathrm{e}-05^{* * *}$ & $2.26 \mathrm{e}-05^{5^{* * *}}$ & $2.27 e-05^{* * *}$ \\
\hline & (11.25) & (11.01) & (11.30) & (11.33) & (11.37) \\
\hline \multirow[t]{2}{*}{ PCM } & $-0.888^{* * *}$ & $-0.904^{* * *}$ & $-0.873^{* * *}$ & $-0.873^{* * *}$ & $-0.875^{* * *}$ \\
\hline & $(-6.49)$ & $(-6.59)$ & $(-6.38)$ & $(-6.36)$ & $(-6.39)$ \\
\hline \multirow{2}{*}{ INTANGTA } & $-1.997^{* * *}$ & $-1.991^{* * *}$ & $-2.023^{* * *}$ & $-2.031^{* * *}$ & $-2.038^{* * *}$ \\
\hline & $(-7.64)$ & $(-7.65)$ & $(-7.74)$ & $(-7.78)$ & $(-7.81)$ \\
\hline \multirow[t]{2}{*}{$\mathrm{CF}$} & $0.0258^{* * *}$ & $0.0262^{* * *}$ & $0.0257^{* * *}$ & $0.0258^{* * *}$ & $0.0259^{* * *}$ \\
\hline & (3.47) & $(3.53)$ & (3.44) & $(3.45)$ & (3.47) \\
\hline \multirow[t]{2}{*}{ CFSTOCK } & $-0.0108^{* * * *}$ & $-0.0108^{* * * *}$ & $-0.0107^{* * * *}$ & $-0.0106^{* * *}$ & $-0.0105^{* * *}$ \\
\hline & $(-6.59)$ & $(-6.64)$ & $(-6.50)$ & $(-6.44)$ & $(-6.40)$ \\
\hline \multirow{2}{*}{ LDR } & $0.0146^{* * *}$ & $0.0133^{* * *}$ & $0.0141^{* * *}$ & $0.0140^{* * *}$ & $0.0141^{* * *}$ \\
\hline & $(4.52)$ & $(4.11)$ & $(4.34)$ & $(4.33)$ & $(4.37)$ \\
\hline \multirow[t]{2}{*}{ SHRHFA5 } & -0.101 & -0.117 & -0.0848 & -0.0848 & -0.0540 \\
\hline & $(-1.06)$ & $(-1.23)$ & $(-0.89)$ & $(-0.89)$ & $(-0.57)$ \\
\hline \multirow{2}{*}{ _cons } & $-2.082^{* * *}$ & $-2.036^{* * *}$ & $-1.941^{* * * *}$ & $-1.942^{* * *}$ & $-1.654^{* * *}$ \\
\hline & $(-5.46)$ & $(-5.34)$ & $(-5.01)$ & $(-5.04)$ & $(-4.14)$ \\
\hline & 13764 & 13764 & 13729 & 13745 & 13764 \\
\hline adj. $R^{2}$ & 0.499 & 0.500 & 0.499 & 0.499 & 0.499 \\
\hline
\end{tabular}

Panel B. Innovation measured by RDIN

\begin{tabular}{llllll}
\hline & $(1)$ & $(2)$ & $(3)$ & $(4)$ & $(5)$ \\
& ZERO & LIU & GAM & AMIHUD & AMIHUDSR \\
\hline LIQ & $-0.0568^{* * *}$ & $-9.45 \mathrm{e}-06$ & $-0.00270^{* *}$ & $-0.00188^{* * *}$ & $-0.0819^{* * *}$ \\
& $(-3.91)$ & $(-1.37)$ & $(-2.05)$ & $(-3.92)$ & $(-3.97)$ \\
LNAGE & $-0.00971^{* * * *}$ & $-0.00989^{* * *}$ & $-0.00999^{* * *}$ & $-0.0101^{* * *}$ & $-0.0100^{* * *}$ \\
& $(-11.54)$ & $(-11.69)^{* * *}$ & $(-11.76)$ & $(-11.94)$ & $(-11.88)$ \\
CAPEXTA & $0.0503^{* * *}$ & $0.0520^{* * *}$ & $0.0517^{* * *}$ & $0.0522^{* * *}$ & $0.0520^{* * *}$ \\
& $(8.51)$ & $(8.81)$ & $(8.77)$ & $(8.85)$ & $(8.83)$ \\
SIZE & $-0.00125^{* * * *}$ & $-0.00139^{* * *}$ & $-0.00147^{* * *}$ & $-0.00155^{* * *}$ & $-0.00196^{* * *}$ \\
& $(-3.02)$ & $(-3.38)$ & $(-3.56)$ & $(-3.77)$ & $(-4.62)$ \\
ROE & $-0.0174^{* * *}$ & $-0.0167^{* * *}$ & $-0.0165^{* * *}$ & $-0.0162^{* * *}$ & $-0.0164^{* * *}$ \\
& $(-5.44)$ & $(-5.23)$ & $(-5.18)$ & $(-5.10)$ & $(-5.15)$ \\
Q & $0.00234^{* * *}$ & $0.00240^{* * *}$ & $0.00241^{* * *}$ & $0.00251^{* * *}$ & $0.00238^{* * *}$
\end{tabular}




\begin{tabular}{|c|c|c|c|c|c|}
\hline & $(9.38)$ & (9.59) & $(9.60)$ & (9.84) & $(9.53)$ \\
\hline PPETA & $\begin{array}{l}-0.0188^{* * * *} \\
(-9.35)\end{array}$ & $\begin{array}{l}-0.0199^{* * * *} \\
(-9.99)\end{array}$ & $\begin{array}{l}-0.0197^{* * * *} \\
(-9.91)\end{array}$ & $\begin{array}{l}-0.0196^{* * * *} \\
(-9.84)\end{array}$ & $\begin{array}{l}-0.0195^{* * * *} \\
(-9.80)\end{array}$ \\
\hline MSHARE & $\begin{array}{l}0.0148^{* * * *} \\
(6.46)\end{array}$ & $\begin{array}{l}0.0150^{* * *} \\
(6.59)\end{array}$ & $\begin{array}{l}0.0154^{* * *} \\
(6.72)\end{array}$ & $\begin{array}{l}0.0157^{* * *} \\
(6.85)\end{array}$ & $\begin{array}{l}0.0158^{* * * *} \\
(6.90)\end{array}$ \\
\hline MPAY & $\begin{array}{l}0.00697^{* * *} \\
(16.05)\end{array}$ & $\begin{array}{l}0.00709^{* * *} \\
(16.30)\end{array}$ & $\begin{array}{l}0.00711^{* * *} \\
(16.31)\end{array}$ & $\begin{array}{l}0.00707^{* * *} \\
(16.24)\end{array}$ & $\begin{array}{l}0.00704^{* * *} \\
(16.18)\end{array}$ \\
\hline COMPETITION & $\begin{array}{l}0.0436^{* * *} \\
(9.72)\end{array}$ & $\begin{array}{l}0.0436^{* * *} \\
(9.69)\end{array}$ & $\begin{array}{l}0.0436^{* * *} \\
(9.70)\end{array}$ & $\begin{array}{l}0.0426^{* * *} \\
(9.46)\end{array}$ & $\begin{array}{l}0.0428^{* * *} \\
(9.51)\end{array}$ \\
\hline EMPLOYEE & $\begin{array}{l}-1.10 \mathrm{e}-07^{* * *} \\
(-2.62)\end{array}$ & $\begin{array}{l}-1.25 \mathrm{e}-07^{* * *} \\
(-2.97)\end{array}$ & $\begin{array}{l}-1.19 \mathrm{e}-07^{* * *} \\
(-2.83)\end{array}$ & $\begin{array}{l}-1.12 \mathrm{e}-07^{* * *} \\
(-2.66)\end{array}$ & $\begin{array}{l}-1.06 \mathrm{e}-07^{* *} \\
(-2.52)\end{array}$ \\
\hline PCM & $\begin{array}{l}-0.0250^{* * *} \\
(-5.05)\end{array}$ & $\begin{array}{l}-0.0249^{\text {**** }} \\
(-5.02)\end{array}$ & $\begin{array}{l}-0.0248^{* * *} \\
(-4.99)\end{array}$ & $\begin{array}{l}-0.0246^{* * *} \\
(-4.97)\end{array}$ & $\begin{array}{l}-0.0246^{* * *} \\
(-4.96)\end{array}$ \\
\hline INTANGTA & $\begin{array}{l}0.00497 \\
(0.73)\end{array}$ & $\begin{array}{l}0.00443 \\
(0.65)\end{array}$ & $\begin{array}{l}0.00425 \\
(0.62)\end{array}$ & $\begin{array}{l}0.00374 \\
(0.55)\end{array}$ & $\begin{array}{l}0.00343 \\
(0.50)\end{array}$ \\
\hline $\mathrm{CF}$ & $\begin{array}{l}0.000445^{* *} \\
(1.98)\end{array}$ & $\begin{array}{l}0.000450^{* *} \\
(2.01)\end{array}$ & $\begin{array}{l}0.000449^{* *} \\
(2.00)\end{array}$ & $\begin{array}{l}0.000452^{* *} \\
(2.01)\end{array}$ & $\begin{array}{l}0.000449^{* * *} \\
(2.00)\end{array}$ \\
\hline CFSTOCK & $\begin{array}{l}0.000126^{*} \\
(1.95)\end{array}$ & $\begin{array}{l}0.000127^{*} \\
(1.96)\end{array}$ & $\begin{array}{l}0.000130^{* * *} \\
(1.99)\end{array}$ & $\begin{array}{l}0.000134^{* *} \\
(2.06)\end{array}$ & $\begin{array}{l}0.000136^{* *} \\
(2.10)\end{array}$ \\
\hline LDR & $\begin{array}{l}0.00278^{* * *} \\
(16.61)\end{array}$ & $\begin{array}{l}0.00276^{* * *} \\
(16.50)\end{array}$ & $\begin{array}{l}0.00276^{* * *} \\
(16.51)\end{array}$ & $\begin{array}{l}0.00276^{* * *} \\
(16.50)\end{array}$ & $\begin{array}{l}0.00276^{* * *} \\
(16.55)\end{array}$ \\
\hline SHRHFA5 & $\begin{array}{l}-0.0213^{* * *} \\
(-9.38)\end{array}$ & $\begin{array}{l}-0.0212^{* * *} \\
(-9.37)\end{array}$ & $\begin{array}{l}-0.0210^{\text {*** }} \\
(-9.25)\end{array}$ & $\begin{array}{l}-0.0207^{* * *} \\
(-9.13)\end{array}$ & $\begin{array}{l}-0.0195^{* * *} \\
(-8.54)\end{array}$ \\
\hline _cons & $\begin{array}{l}-0.0506^{* * *} \\
(-5.25)\end{array}$ & $\begin{array}{l}-0.0503^{* * *} \\
(-5.22)\end{array}$ & $\begin{array}{l}-0.0484^{* * *} \\
(-4.97)\end{array}$ & $\begin{array}{l}-0.0466^{* * *} \\
(-4.83)\end{array}$ & $\begin{array}{l}-0.0353^{* * *} \\
(-3.49)\end{array}$ \\
\hline$N$ & 13771 & 13771 & 13736 & 13752 & 13771 \\
\hline adj. $R^{2}$ & 0.401 & 0.401 & 0.401 & 0.401 & 0.401 \\
\hline
\end{tabular}

$t$ statistics in parentheses, ${ }^{*} p<0.1,{ }^{* *} p<0.05,{ }^{* * * *} p<0.01$

Table 3. Stock liquidity and firm innovation (FE specifications)

Panel A. Innovation measured by LNRD

\begin{tabular}{|c|c|c|c|c|c|}
\hline & (1) & (2) & (3) & (4) & (5) \\
\hline & ZERO & LIU & GAM & AMIHUD & AMIHUDSR \\
\hline \multirow[t]{2}{*}{ LIQ } & 0.849 & $-6.01 \mathrm{e}-04^{* * *}$ & $-0.104^{* * *}$ & $-0.0246^{* *}$ & -0.814 \\
\hline & (1.49) & $(-3.76)$ & $(-3.14)$ & $(-2.31)$ & $(-1.37)$ \\
\hline \multirow[t]{2}{*}{ LNAGE } & $-0.664^{* * *}$ & $-0.650^{* * *}$ & $-0.680^{* * *}$ & $-0.674^{* * *}$ & $-0.658^{* * *}$ \\
\hline & $(-4.30)$ & $(-4.24)$ & $(-4.42)$ & $(-4.38)$ & $(-4.27)$ \\
\hline \multirow[t]{2}{*}{ CAPEXTA } & $0.768^{* * *}$ & $0.718^{* * * *}$ & $0.743^{* * *}$ & $0.738^{* * *}$ & $0.748^{* * *}$ \\
\hline & $(3.40)$ & (3.18) & $(3.28)$ & $(3.26)$ & $(3.31)$ \\
\hline \multirow[t]{2}{*}{ SIZE } & $0.607^{* * * *}$ & $0.610^{* * * *}$ & $0.604^{* * * *}$ & $0.606^{* * * *}$ & $0.602^{* * * *}$ \\
\hline & $(14.47)$ & $(14.52)$ & $(14.40)$ & $(14.43)$ & $(14.36)$ \\
\hline \multirow[t]{2}{*}{ ROE } & $0.400^{* * * *}$ & $0.390^{* * * *}$ & $0.389^{* * *}$ & $0.392^{* * * *}$ & $0.391^{* * * *}$ \\
\hline & $(3.31)$ & $(3.23)$ & $(3.23)$ & $(3.25)$ & $(3.24)$ \\
\hline \multirow[t]{2}{*}{ Q } & $0.0125^{*}$ & $0.0152^{* *}$ & 0.0121 & $0.0124^{*}$ & 0.0113 \\
\hline & (1.69) & $(2.05)$ & $(1.61)$ & $(1.65)$ & $(1.52)$ \\
\hline \multirow[t]{2}{*}{ PPETA } & $0.345^{* *}$ & $0.348^{* *}$ & $0.347^{* *}$ & $0.346^{* *}$ & $0.351^{* *}$ \\
\hline & (2.08) & (2.11) & (2.10) & (2.09) & $(2.12)$ \\
\hline \multirow[t]{2}{*}{ MSHARE } & $0.541^{* * *}$ & $0.535^{\text {*** }}$ & $0.555^{* * *}$ & $0.552^{* * *}$ & $0.544^{* * *}$ \\
\hline & $(4.12)$ & $(4.08)$ & $(4.26)$ & $(4.23)$ & (4.13) \\
\hline \multirow[t]{2}{*}{ MPAY } & $0.0993^{* * *}$ & $0.0969^{* * *}$ & $0.0956^{* * *}$ & $0.0962^{* * *}$ & $0.0957^{* * *}$ \\
\hline & $(2.74)$ & $(2.68)$ & $(2.64)$ & $(2.66)$ & $(2.65)$ \\
\hline \multirow[t]{2}{*}{ COMPETITION } & $1.176^{\text {*** }}$ & $1.153^{* * *}$ & $1.159^{* * *}$ & $1.167^{* * *}$ & $1.168^{* * *}$ \\
\hline & $(3.71)$ & (3.67) & $(3.67)$ & $(3.69)$ & $(3.70)$ \\
\hline \multirow[t]{2}{*}{ EMPLOYEE } & $2.58 \mathrm{e}-05^{* * *}$ & $2.53 \mathrm{e}-05^{* * *}$ & $2.6 \mathrm{e}-05^{* * *}$ & $2.59 \mathrm{e}-05^{* * *}$ & $2.6 \mathrm{e}-05^{* * *}$ \\
\hline & $(4.11)$ & $(4.02)$ & $(4.12)$ & $(4.12)$ & $(4.12)$ \\
\hline \multirow[t]{2}{*}{ PCM } & 0.0781 & 0.0503 & 0.0764 & 0.0768 & 0.0771 \\
\hline & $(0.46)$ & $(0.30)$ & $(0.45)$ & $(0.45)$ & $(0.45)$ \\
\hline
\end{tabular}




\begin{tabular}{llllll} 
INTANGTA & -0.0959 & -0.0722 & -0.0768 & -0.0702 & -0.0779 \\
& $(-0.23)$ & $(-0.18)$ & $(-0.19)$ & $(-0.17)$ & $(-0.19)$ \\
CF & 0.0102 & 0.0103 & 0.0102 & 0.0101 & 0.0101 \\
& $(1.62)$ & $(1.64)$ & $(1.62)$ & $(1.60)$ & $(1.61)$ \\
CFSTOCK & -0.00249 & -0.00253 & -0.00250 & -0.00245 & -0.00244 \\
& $(-1.36)$ & $(-1.40)$ & $(-1.37)$ & $(-1.34)$ & $(-1.34)$ \\
LDR & $0.00983^{* *}$ & $0.00937^{* *}$ & $0.00970^{* *}$ & $0.00963^{* *}$ & $0.00984^{* *}$ \\
& $(2.18)$ & $(2.08)$ & $(2.14)$ & $(2.13)$ & $(2.18)$ \\
SHRHFA5 & -0.0567 & -0.0628 & -0.0545 & -0.0612 & -0.0442 \\
& $(-0.19)$ & $(-0.21)$ & $(-0.19)$ & $(-0.21)$ & $(-0.15)$ \\
_cons & $2.115^{* *}$ & $2.092^{* *}$ & $2.302^{* *}$ & $2.225^{* *}$ & $2.297^{* *}$ \\
& $(2.10)$ & $(2.06)$ & $(2.27)$ & $(2.19)$ & $(2.26)$ \\
\hline$N$ & 13764 & 13764 & 13729 & 13745 & 13764 \\
adj. $R^{2}$ & 0.501 & 0.502 & 0.502 & 0.501 & 0.501 \\
\hline
\end{tabular}

Panel B. Innovation measured by RDIN

\begin{tabular}{|c|c|c|c|c|c|}
\hline & (1) & (2) & (3) & (4) & (5) \\
\hline & ZERO & LIU & GAM & AMIHUD & AMIHUDSR \\
\hline LIQ & $\begin{array}{l}-0.00333 \\
(-0.27)\end{array}$ & $\begin{array}{l}-5.84 \mathrm{e}-06 \\
(-1.42)\end{array}$ & $\begin{array}{l}-0.00158 \\
(-1.56)\end{array}$ & $\begin{array}{l}-0.00103^{* * * *} \\
(-3.13)\end{array}$ & $\begin{array}{l}-0.0350^{* * *} \\
(-199)\end{array}$ \\
\hline LNAGE & $\begin{array}{l}-0.00572 \\
(-1.30)\end{array}$ & $\begin{array}{l}-0.00566 \\
(-1.29)\end{array}$ & $\begin{array}{l}-0.00587 \\
(-1.33)\end{array}$ & $\begin{array}{l}-0.00580 \\
(-1.31)\end{array}$ & $\begin{array}{l}-0.00572 \\
(-1.30)\end{array}$ \\
\hline CAPEXTA & $\begin{array}{l}0.0378^{* * *} \\
(5.73)\end{array}$ & $\begin{array}{l}0.0375^{* * *} \\
(5.68)\end{array}$ & $\begin{array}{l}0.0381^{* * *} \\
(5.73)\end{array}$ & $\begin{array}{l}0.0377^{* * *} \\
(5.69)\end{array}$ & $\begin{array}{l}0.0377^{* * *} \\
(5.70)\end{array}$ \\
\hline SIZE & $\begin{array}{l}-0.000301 \\
(-0.30)\end{array}$ & $\begin{array}{l}-0.000259 \\
(-0.25)\end{array}$ & $\begin{array}{l}-0.000302 \\
(-0.30)\end{array}$ & $\begin{array}{l}-0.000300 \\
(-0.29)\end{array}$ & $\begin{array}{l}-0.000459 \\
(-0.45)\end{array}$ \\
\hline ROE & $\begin{array}{l}0.00547^{*} \\
(1.70)\end{array}$ & $\begin{array}{l}0.00548^{*} \\
(1.70)\end{array}$ & $\begin{array}{l}0.00543^{*} \\
(1.68)\end{array}$ & $\begin{array}{l}0.00553^{*} \\
(1.71)\end{array}$ & $\begin{array}{l}0.00547^{*} \\
(1.70)\end{array}$ \\
\hline Q & $\begin{array}{l}0.000756^{* * * *} \\
(2.67)\end{array}$ & $\begin{array}{l}0.000788^{* * *} \\
(2.73)\end{array}$ & $\begin{array}{l}0.000768^{* * *} \\
(2.67)\end{array}$ & $\begin{array}{l}0.000786^{* * *} \\
(2.73)\end{array}$ & $\begin{array}{l}0.000723^{* *} \\
(2.54)\end{array}$ \\
\hline PPETA & $\begin{array}{l}0.00342 \\
(0.86)\end{array}$ & $\begin{array}{l}0.00339 \\
(0.85)\end{array}$ & $\begin{array}{l}0.00346 \\
(0.87)\end{array}$ & $\begin{array}{l}0.00343 \\
(0.86)\end{array}$ & $\begin{array}{l}0.00344 \\
(0.87)\end{array}$ \\
\hline MSHARE & $\begin{array}{l}0.00775^{*} \\
(1.83)\end{array}$ & $\begin{array}{l}0.00768^{*} \\
(1.82)\end{array}$ & $\begin{array}{l}0.00785^{*} \\
(1.86)\end{array}$ & $\begin{array}{l}0.00787^{*} \\
(1.86)\end{array}$ & $\begin{array}{l}0.00788^{*} \\
(1.86)\end{array}$ \\
\hline MPAY & $\begin{array}{l}0.00262^{* * *} \\
(2.96)\end{array}$ & $\begin{array}{l}0.00263^{* * *} \\
(2.98)\end{array}$ & $\begin{array}{l}0.00261^{* * *} \\
(2.95)\end{array}$ & $\begin{array}{l}0.00260^{* * *} \\
(2.94)\end{array}$ & $\begin{array}{l}0.00259^{* * *} \\
(2.93)\end{array}$ \\
\hline COMPETITION & $\begin{array}{l}0.0720^{* * * *} \\
(5.35)\end{array}$ & $\begin{array}{l}0.0718^{* * * *} \\
(5.35)\end{array}$ & $\begin{array}{l}0.0714^{* * * *} \\
(5.31)\end{array}$ & $\begin{array}{l}0.0716^{* * *} \\
(5.33)\end{array}$ & $\begin{array}{l}0.0718^{* * *} \\
(5.34)\end{array}$ \\
\hline EMPLOYEE & $\begin{array}{l}-1.14 \mathrm{e}-07 \\
(-1.10)\end{array}$ & $\begin{array}{l}-1.19 \mathrm{e}-07 \\
(-1.15)\end{array}$ & $\begin{array}{l}-1.13 e-07 \\
(-1.08)\end{array}$ & $\begin{array}{l}-1.12 \mathrm{e}-07 \\
(-1.08)\end{array}$ & $\begin{array}{l}-1.09 \mathrm{e}-07 \\
(-1.05)\end{array}$ \\
\hline PCM & $\begin{array}{l}-0.0462^{* * * *} \\
(-7.25)\end{array}$ & $\begin{array}{l}-0.0464^{* * *} \\
(-7.30)\end{array}$ & $\begin{array}{l}-0.0463^{* * *} \\
(-7.27)\end{array}$ & $\begin{array}{l}-0.0462^{* * *} \\
(-7.26)\end{array}$ & $\begin{array}{l}-0.0462^{* * * *} \\
(-7.25)\end{array}$ \\
\hline INTANGTA & $\begin{array}{l}0.0183 \\
(1.43)\end{array}$ & $\begin{array}{l}0.0183 \\
(1.44)\end{array}$ & $\begin{array}{l}0.0183 \\
(1.43)\end{array}$ & $\begin{array}{l}0.0184 \\
(1.44)\end{array}$ & $\begin{array}{l}0.0183 \\
(1.44)\end{array}$ \\
\hline $\mathrm{CF}$ & $\begin{array}{l}0.000256 \\
(1.32)\end{array}$ & $\begin{array}{l}0.000258 \\
(1.34)\end{array}$ & $\begin{array}{l}0.000254 \\
(1.31)\end{array}$ & $\begin{array}{l}0.000258 \\
(1.34)\end{array}$ & $\begin{array}{l}0.000258 \\
(1.33)\end{array}$ \\
\hline CFSTOCK & $\begin{array}{l}-0.00000883 \\
(-0.11)\end{array}$ & $\begin{array}{l}-0.00000949 \\
(-0.12)\end{array}$ & $\begin{array}{l}-0.0000100 \\
(-0.13)\end{array}$ & $\begin{array}{l}-0.00000758 \\
(-0.10)\end{array}$ & $\begin{array}{l}-0.00000761 \\
(-0.10)\end{array}$ \\
\hline LDR & $\begin{array}{l}0.00120^{* * *} \\
(5.91)\end{array}$ & $\begin{array}{l}0.00120^{* * *} \\
(5.89)\end{array}$ & $\begin{array}{l}0.00120^{* * * *} \\
(5.89)\end{array}$ & $\begin{array}{l}0.00121^{* * *} \\
(5.92)\end{array}$ & $\begin{array}{l}0.00121^{* * *} \\
(5.93)\end{array}$ \\
\hline SHRHFA5 & $\begin{array}{l}-0.00274 \\
(-0.44)\end{array}$ & $\begin{array}{l}-0.00276 \\
(-0.45)\end{array}$ & $\begin{array}{l}-0.00268 \\
(-0.43)\end{array}$ & $\begin{array}{l}-0.00261 \\
(-0.42)\end{array}$ & $\begin{array}{l}-0.00208 \\
(-0.33)\end{array}$ \\
\hline _cons & $\begin{array}{l}-0.00816 \\
(-0.31)\end{array}$ & $\begin{array}{l}-0.00923 \\
(-0.35)\end{array}$ & $\begin{array}{l}-0.00747 \\
(-0.28)\end{array}$ & $\begin{array}{l}-0.00765 \\
(-0.29)\end{array}$ & $\begin{array}{l}-0.00324 \\
(-0.12)\end{array}$ \\
\hline $\begin{array}{l}N \\
\text { adi } R^{2}\end{array}$ & $\begin{array}{l}13771 \\
0.174\end{array}$ & $\begin{array}{l}13771 \\
0.174\end{array}$ & $\begin{array}{l}13736 \\
0.174\end{array}$ & $\begin{array}{l}13752 \\
0.174\end{array}$ & $\begin{array}{l}13771 \\
0.174\end{array}$ \\
\hline
\end{tabular}

$\mathrm{t}$ statistics in parentheses, $* \mathrm{p}<0.1, * * \mathrm{p}<0.05, * * * \mathrm{p}<0.01$ 


\section{Additional Evidence and Robustness Tests}

While the tests in section 4 are suggestive of a role for stock liquidity in the firm innovation, they are likely suffering from endogeneity concern that results from self-selection bias. In this section, I try to address this concern by applying the Heckman two-stage model. In addition, I run several robustness checks.

\subsection{Heckman Two-stage Model}

In this subsection, I examine whether my results are robust after correcting for the possible endogeneity that results from self-selection bias by applying the Heckman two-stage model.

The data of $R \& D$ expenditures is limited to those firms that have disclosed their R\&D expenditures. Thus, the estimation results may only suggest the correlation between firm innovation and stock liquidity in those firms. Therefore, in order to control the estimation bias resulted from sample selection bias, I use the Heckman two-stage regression model.

The Heckman two-stage regression model includes two regression equations, one is the principal equation which is for the estimation of the correlation between stock liquidity and firm innovation, and the other one is the equation for sample selection. The sample selection equation is used to estimate the possibility of firm's disclosure of R\&D expenditures and its dependent variable is a dummy variable IFSEE (whether the firm disclose R\&D expenditures). In addition to all the control variables in the main equation, there is one more control variable in the sample selection equation: a dummy variable HIGHTECH (whether a firm belongs to the high technology industry). I choose the maximum likelihood estimation approach (MLE) in my regression.

Table 4 presents the results when the dependent variable is LNRD. The last row of the table reports the results of Wald test. It can be seen that there indeed exists sample selection bias. The coefficients on LIU, GAM, AMIHUD and AMIHUDSR are negative and both economically and statistically significant. This suggests that the positive relationship between stock liquidity and firm innovation is still robust even after controlling self-selection bias. Because the table is too long, the regression result of the model which uses RDIN as the dependent variable is not reported here. The regression result is consistent with the regression result of the model which uses LNRD as the dependent variable.

\subsection{Robustness Tests}

In order to check the reliability of my study findings, I run several robustness tests. Firstly, I use the ratio of R\&D expenditures to total asset (RDTA) as an alternative measure of firm innovation. Secondly, I delete control variables such as industry, and year variables. Thirdly, I take regression analysis by using a different sample. My study period in section 4 and section 5 is from 2006 to 2016 which includes several major events such as the reform of the shareholder structure of the Chinese market, reform of stock market stamp tax and global financial crisis. In order to reduce the potential bias, in this robustness test I study the period from 2009 to 2016. The alternative regression results (not presented) show that the primary study findings do not change substantially. Thus, my study results are robust in general.

Table 4. Stock liquidity and firm innovation (Heckman model)

\begin{tabular}{llllll}
\hline & $(1)$ & $(2)$ & $(3)$ & $(4)$ & $(5)$ \\
& ZERO & LIU & GAM & AMIHUD & AMIHUDSR \\
\hline LNRD & & & & & \\
LIQ & -0.944 & $-0.00106^{* * *}$ & $-0.0729^{* *}$ & $-0.0363^{* * *}$ & $-1.072^{* *}$ \\
& $(-1.44)$ & $(-4.72)$ & $(-2.24)$ & $(-3.24)$ & $(-1.98)$ \\
LNAGE & $-0.395^{* * *}$ & $-0.400^{* * *}$ & $-0.403^{* * *}$ & $-0.404^{* * *}$ & $-0.399^{* * *}$ \\
& $(-12.05)$ & $(-12.19)$ & $(-12.20)$ & $(-12.27)$ & $(-12.15)$ \\
CAPEXTA & $0.699^{* * *}$ & $0.705^{* * *}$ & $0.721^{* * *}$ & $0.726^{* * *}$ & $0.731^{* * * *}$ \\
& $(2.68)$ & $(2.72)$ & $(2.77)$ & $(2.79)$ & $(2.81)$ \\
SIZE & $0.545^{* * *}$ & $0.544^{* * *}$ & $0.538^{* * *}$ & $0.539^{* * *}$ & $0.536^{* * *}$ \\
& $(30.45)$ & $(30.58)$ & $(29.89)$ & $(30.06)$ & $(29.13)$ \\
ROE & $1.593^{* * *}$ & $1.583^{* * *}$ & $1.610^{* * *}$ & $1.614^{* * *}$ & $1.608^{* * *}$
\end{tabular}




\begin{tabular}{|c|c|c|c|c|c|}
\hline \multirow{3}{*}{ Q } & (10.66) & (10.61) & $(10.81)$ & (10.83) & (10.80) \\
\hline & $-0.0280^{* * * *}$ & $-0.0240^{* * * *}$ & $-0.0274^{* * *}$ & $-0.0252^{* * *}$ & $-0.0274^{* * *}$ \\
\hline & $(-4.12)$ & $(-3.52)$ & $(-3.99)$ & $(-3.60)$ & $(-4.03)$ \\
\hline \multirow[t]{2}{*}{ PPETA } & $-0.345^{* * * *}$ & $-0.373^{* * * *}$ & $-0.368^{* * * *}$ & $-0.366^{* * * *}$ & $-0.362^{* * * *}$ \\
\hline & $(-3.13)$ & $(-3.41)$ & $(-3.36)$ & $(-3.33)$ & $(-3.30)$ \\
\hline \multirow[t]{2}{*}{ MSHARE } & $-0.692^{* * * *}$ & $-0.683^{* * *}$ & $-0.676^{* * * *}$ & $-0.670^{* * * *}$ & $-0.674^{* * * *}$ \\
\hline & $(-7.86)$ & $(-7.71)$ & $(-7.56)$ & $(-7.45)$ & $(-7.47)$ \\
\hline \multirow[t]{2}{*}{ MPAY } & $0.167^{* * *}$ & $0.169^{* * *}$ & $0.170^{* * *}$ & $0.170^{* * *}$ & $0.170^{* * *}$ \\
\hline & $(6.54)$ & $(6.60)$ & $(6.61)$ & $(6.59)$ & $(6.57)$ \\
\hline \multirow[t]{2}{*}{ COMPETITION } & -0.180 & -0.195 & -0.185 & -0.201 & -0.189 \\
\hline & $(-1.30)$ & $(-1.42)$ & $(-1.34)$ & $(-1.46)$ & $(-1.37)$ \\
\hline \multirow[t]{2}{*}{ EMPLOYEE } & $1.84 \mathrm{e}-05^{* * *}$ & $1.79 \mathrm{e}-05^{* * *}$ & $1.85 \mathrm{e}-05^{* * *}$ & $1.85 \mathrm{e}-05^{* * *}$ & $1.84 \mathrm{e}-05^{* * *}$ \\
\hline & $(8.31)$ & $(8.14)$ & $(8.34)$ & $(8.35)$ & $(8.31)$ \\
\hline \multirow[t]{2}{*}{ PCM } & $-0.915^{* * *}$ & $-0.934^{* * *}$ & $-0.904^{* * *}$ & $-0.906^{* * * *}$ & $-0.908^{* * * *}$ \\
\hline & $(-6.20)$ & $(-6.34)$ & $(-6.11)$ & $(-6.12)$ & $(-6.14)$ \\
\hline \multirow[t]{2}{*}{ INTANGTA } & $-1.968^{* * *}$ & $-1.958^{* * *}$ & $-1.988^{* * *}$ & $-1.992^{* * *}$ & $-1.991^{* * *}$ \\
\hline & $(-7.00)$ & $(-6.97)$ & $(-7.06)$ & $(-7.08)$ & $(-7.08)$ \\
\hline \multirow[t]{2}{*}{$\mathrm{CF}$} & $0.0192^{* *}$ & $0.0196^{* *}$ & $0.0192^{* *}$ & $0.0193^{* *}$ & $0.0193^{* *}$ \\
\hline & $(2.38)$ & $(2.45)$ & $(2.37)$ & $(2.38)$ & $(2.39)$ \\
\hline \multirow[t]{2}{*}{ CFSTOCK } & 0.00338 & 0.00329 & $0.00350^{*}$ & $0.00348^{*}$ & $0.00346^{*}$ \\
\hline & $(1.62)$ & $(1.58)$ & $(1.66)$ & $(1.65)$ & $(1.65)$ \\
\hline \multirow[t]{2}{*}{ LDR } & $-0.0258^{* * * *}$ & $-0.0268^{* * *}$ & $-0.0263^{* * *}$ & $-0.0261^{* * *}$ & $-0.0259^{* * * *}$ \\
\hline & $(-5.82)$ & $(-6.06)$ & $(-5.91)$ & $(-5.86)$ & $(-5.83)$ \\
\hline \multirow[t]{2}{*}{ SHRHFA5 } & 0.0660 & 0.0489 & 0.0812 & 0.0765 & 0.0893 \\
\hline & $(0.63)$ & $(0.46)$ & $(0.77)$ & $(0.73)$ & $(0.85)$ \\
\hline \multirow[t]{2}{*}{ _cons } & $2.852^{* * *}$ & $2.870^{* * *}$ & $2.967^{* * *}$ & $2.939^{* * *}$ & $3.037^{* * *}$ \\
\hline & $(5.44)$ & $(5.46)$ & $(5.62)$ & $(5.56)$ & $(5.78)$ \\
\hline \multicolumn{6}{|l|}{ IFSEE } \\
\hline \multirow[t]{2}{*}{ LNAGE } & $0.220^{* * *}$ & $0.221^{* * *}$ & $0.221^{* * *}$ & $0.222^{* * *}$ & $0.220^{* * *}$ \\
\hline & $(7.61)$ & $(7.61)$ & $(7.63)$ & $(7.64)$ & $(7.61)$ \\
\hline \multirow[t]{2}{*}{ CAPEXTA } & $1.035^{* * *}$ & $1.040^{* * *}$ & $1.049^{* * *}$ & $1.051^{* * *}$ & $1.038^{* * *}$ \\
\hline & $(4.98)$ & $(5.00)$ & $(5.04)$ & $(5.05)$ & (4.99) \\
\hline \multirow[t]{2}{*}{ SIZE } & $0.157^{* * *}$ & $0.158^{* * *}$ & $0.159^{* * *}$ & $0.158^{* * *}$ & $0.157^{* * *}$ \\
\hline & (11.55) & (11.56) & (11.67) & (11.63) & (11.55) \\
\hline \multirow[t]{2}{*}{ ROE } & $-0.521^{* * *}$ & $-0.522^{* * *}$ & $-0.524^{* * *}$ & $-0.523^{* * *}$ & $-0.521^{* * *}$ \\
\hline & $(-5.51)$ & $(-5.51)$ & $(-5.54)$ & $(-5.53)$ & $(-5.51)$ \\
\hline \multirow[t]{2}{*}{$\mathrm{Q}$} & $0.0692^{* * * *}$ & $0.0699^{* * * *}$ & $0.0698^{* * * *}$ & $0.0695^{* * *}$ & $0.0691^{* * *}$ \\
\hline & $(10.51)$ & (10.59) & (10.58) & (10.53) & $(10.50)$ \\
\hline \multirow[t]{2}{*}{ PPETA } & $-0.302^{* * *}$ & $-0.302^{* * *}$ & $-0.302^{* * * *}$ & $-0.303^{* * *}$ & $-0.303^{* * *}$ \\
\hline & $(-4.47)$ & $(-4.48)$ & $(-4.48)$ & $(-4.50)$ & $(-4.49)$ \\
\hline \multirow[t]{2}{*}{ MSHARE } & $2.888^{* * * *}$ & $2.900^{* * * *}$ & $2.884^{* * * *}$ & $2.890^{* * * *}$ & $2.895^{* * *}$ \\
\hline & $(13.41)$ & $(13.40)$ & (13.33) & (13.28) & (13.29) \\
\hline
\end{tabular}




\begin{tabular}{|c|c|c|c|c|c|}
\hline MPAY & $\begin{array}{l}0.272^{* * *} \\
(15.23)\end{array}$ & $\begin{array}{l}0.273^{* * *} \\
(15.23)\end{array}$ & $\begin{array}{l}0.272^{* * *} \\
(15.18)\end{array}$ & $\begin{array}{l}0.273^{* * *} \\
(15.16)\end{array}$ & $\begin{array}{l}0.273^{* * *} \\
(15.16)\end{array}$ \\
\hline \multirow[t]{2}{*}{ COMPETITION } & -0.144 & -0.143 & -0.145 & -0.140 & -0.141 \\
\hline & $(-1.08)$ & $(-1.07)$ & $(-1.08)$ & $(-1.04)$ & $(-1.05)$ \\
\hline \multirow[t]{2}{*}{ EMPLOYEE } & $-7.21 \mathrm{e}-07$ & $-7.89 e-07$ & $-8.5 \mathrm{e}-07$ & $-8.2 \mathrm{e}-07$ & $-7.93 e-07$ \\
\hline & $(-0.40)$ & $(-0.43)$ & $(-0.47)$ & $(-0.45)$ & $(-0.44)$ \\
\hline \multirow[t]{2}{*}{ PCM } & $-0.582^{* * *}$ & $-0.581^{* * *}$ & $-0.586^{* * *}$ & $-0.585^{* * *}$ & $-0.582^{* * *}$ \\
\hline & $(-6.15)$ & $(-6.15)$ & $(-6.18)$ & $(-6.17)$ & $(-6.15)$ \\
\hline \multirow[t]{2}{*}{ INTANGTA } & 0.138 & 0.138 & 0.142 & 0.140 & 0.138 \\
\hline & $(0.72)$ & $(0.72)$ & $(0.74)$ & $(0.73)$ & $(0.72)$ \\
\hline \multirow[t]{2}{*}{$\mathrm{CF}$} & $0.0123^{* * *}$ & $0.0123^{* * *}$ & $0.0121^{* * *}$ & $0.0121^{* * *}$ & $0.0123^{* * *}$ \\
\hline & $(2.74)$ & $(2.76)$ & (2.69) & $(2.70)$ & $(2.74)$ \\
\hline \multirow[t]{2}{*}{ CFSTOCK } & $-0.0239^{* * *}$ & $-0.0240^{* * *}$ & $-0.0241^{* * *}$ & $-0.0241^{* * *}$ & $-0.0240^{* * *}$ \\
\hline & $(-15.56)$ & $(-15.62)$ & $(-15.52)$ & $(-15.50)$ & $(-15.50)$ \\
\hline \multirow[t]{2}{*}{ LDR } & $0.101^{* * *}$ & $0.101^{* * * *}$ & $0.101^{* * * *}$ & $0.101^{* * * *}$ & $0.101^{* * *}$ \\
\hline & (13.04) & (13.02) & (13.04) & (13.01) & (12.99) \\
\hline \multirow[t]{2}{*}{ SHRHFA5 } & 0.0205 & 0.0220 & 0.0134 & 0.0169 & 0.0209 \\
\hline & $(0.23)$ & $(0.25)$ & $(0.15)$ & $(0.19)$ & $(0.24)$ \\
\hline \multirow[t]{2}{*}{ HIGHTECH } & $-0.501^{* * * *}$ & $-0.503^{* * *}$ & $-0.502^{* * * *}$ & $-0.503^{* * * *}$ & $-0.502^{* * * *}$ \\
\hline & $(-13.48)$ & $(-13.43)$ & $(-13.37)$ & $(-13.29)$ & $(-13.30)$ \\
\hline \multirow[t]{2}{*}{ _cons } & $-7.376^{* * *}$ & $-7.393^{* * *}$ & $-7.414^{* * *}$ & $-7.409^{* * *}$ & $-7.381^{* * *}$ \\
\hline & $(-24.65)$ & $(-24.66)$ & $(-24.64)$ & $(-24.62)$ & $(-24.60)$ \\
\hline \multicolumn{6}{|l|}{ athrho } \\
\hline \multirow[t]{2}{*}{ _cons } & $-1.297^{* * *}$ & $-1.289^{* * *}$ & $-1.294^{* * *}$ & $-1.290^{* * *}$ & $-1.291^{* * *}$ \\
\hline & $(-11.43)$ & $(-11.30)$ & $(-11.31)$ & $(-11.16)$ & $(-11.16)$ \\
\hline \multicolumn{6}{|l|}{ lnsigma } \\
\hline \multirow[t]{2}{*}{ _cons } & $0.295^{* * *}$ & $0.292^{* * *}$ & $0.295^{* * * *}$ & $0.294^{* * *}$ & $0.294^{* * *}$ \\
\hline & (13.64) & (13.45) & (13.53) & (13.34) & (13.34) \\
\hline$N$ & 21354 & 21354 & 21319 & 21335 & 21354 \\
\hline $\begin{array}{l}\text { Wald test of } \\
\text { indep. eqns. (rho } \\
=0 \text { ) }\end{array}$ & $\begin{array}{l}\text { Prob > chi2 } \\
=0.0000\end{array}$ & $\begin{array}{l}\text { Prob > chi2 } \\
=0.0000\end{array}$ & $\begin{array}{l}\text { Prob > chi2 } \\
=0.0000\end{array}$ & $\begin{array}{l}\text { Prob > chi2 } \\
=0.0000\end{array}$ & $\begin{array}{l}\text { Prob > chi2 } \\
=0.0000\end{array}$ \\
\hline
\end{tabular}

$\mathrm{t}$ statistics in parentheses, ${ }^{*} \mathrm{p}<0.1,{ }^{* *} \mathrm{p}<0.05,{ }^{* * *} \mathrm{p}<0.01$

\section{Mediating Effects}

The analysis in section 4 and 5 suggest a positive relationship between stock liquidity and R\&D investment. In this section, I will further analyse the hypothesized mechanisms through which stock liquidity may enhance firm innovation according to my theoretical analysis: financing constraints mechanism and agency costs mechanism. It's difficult to provide definitive proof, so my tests are only suggestive. I construct the following mediating effect model to examine the mediating effects of the financing constraints mechanism and the agency costs mechanism.

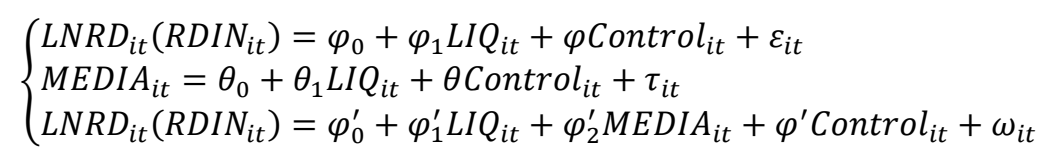


where $L N R D_{i t}$ is the natural logarithm of $\mathrm{R} \& \mathrm{D}$ expenditures, $R D I N_{i t}$ is the ratio of $\mathrm{R} \& \mathrm{D}$ expenditures to revenue, $L I Q_{i t}$ is the stock liquidity of firm i in year $\mathrm{t}$, Control $_{i t}$ are the control variables, $M E D I A_{i t}$ are the mediating variables.

Table 5. Stock liquidity and firm innovation (mediating effect)

\begin{tabular}{|c|c|c|c|c|c|}
\hline \multicolumn{6}{|c|}{ Panel A. Mediating variable LEV - Innovation Measured by LNRD } \\
\hline & ZERO & LIU & GAM & AMIHUD & AMIHUDSR \\
\hline \multirow{2}{*}{$\varphi_{1}$} & $-1.403^{* *}$ & $-0.00113^{* * *}$ & $-0.110^{* * *}$ & $-0.0545^{* * *}$ & $-2.282^{* * * *}$ \\
\hline & $(-1.98)$ & $(-4.87)$ & $(-3.51)$ & $(-4.92)$ & $(-4.38)$ \\
\hline \multirow{2}{*}{$\theta_{1}$} & $0.123^{*}$ & $0.000119^{* * *}$ & $0.0442^{* * *}$ & $0.0178^{* * *}$ & $1.126^{* * *}$ \\
\hline & -1.88 & -4.31 & -9.92 & -11.16 & -16.62 \\
\hline \multirow{2}{*}{$\varphi_{2}^{\prime}$} & $-0.770^{* * *}$ & $-0.764^{* * *}$ & $-0.765^{* * *}$ & $-0.758^{* * *}$ & $-0.755^{* * *}$ \\
\hline & $(-9.61)$ & $(-9.52)$ & $(-9.50)$ & $(-9.41)$ & $(-9.36)$ \\
\hline \multirow{2}{*}{$\varphi_{1}^{\prime}$} & $-1.280^{*}$ & $-0.00107^{* * *}$ & $-0.0860^{* * * *}$ & $-0.0439^{* * *}$ & $-1.633^{* * *}$ \\
\hline & $(-1.82)$ & $(-4.62)$ & $(-2.77)$ & $(-3.99)$ & $(-3.16)$ \\
\hline
\end{tabular}

Panel B. Mediating variable LEV - Innovation Measured by RDIN

\begin{tabular}{|c|c|c|c|c|c|}
\hline$\varphi_{1}$ & $\begin{array}{l}-0.0568^{* * *} \\
(-3.91)\end{array}$ & $\begin{array}{l}-0.00000945 \\
(-1.37)\end{array}$ & $\begin{array}{l}-0.00270^{* *} \\
(-2.05)\end{array}$ & $\begin{array}{l}-0.00188^{* * * *} \\
(-3.92)\end{array}$ & $\begin{array}{l}-0.0819^{* * *} \\
(-3.97)\end{array}$ \\
\hline \multirow{2}{*}{$\theta_{1}$} & $0.123^{*}$ & $0.000119^{* * * *}$ & $0.0442^{* * *}$ & $0.0178^{* * * *}$ & $1.126^{* * * *}$ \\
\hline & -1.88 & -4.31 & -9.92 & -11.16 & -16.62 \\
\hline \multirow{2}{*}{$\varphi_{2}^{\prime}$} & $-0.0132^{* * *}$ & $-0.0133^{* * *}$ & $-0.0132^{* * * *}$ & $-0.0128^{* * *}$ & $-0.0125^{* * *}$ \\
\hline & $(-6.64)$ & $(-6.66)$ & $(-6.62)$ & $(-6.41)$ & $(-6.27)$ \\
\hline \multirow[b]{2}{*}{$\varphi_{1}^{\prime}$} & $-0.0547^{* * *}$ & -0.00000849 & $-0.00229^{*}$ & $-0.00170^{* * *}$ & $-0.0711^{* * *}$ \\
\hline & $(-3.77)$ & $(-1.23)$ & $(-1.74)$ & $(-3.55)$ & $(-3.44)$ \\
\hline
\end{tabular}

Panel C. Mediating variable MCOST - Innovation Measured by LNRD

\begin{tabular}{llllll}
\hline & $-1.403^{* *}$ & $-0.00113^{* * *}$ & $-0.110^{* * *}$ & $-0.0545^{* * *}$ & $-2.282^{* * *}$ \\
& $(-1.98)$ & $(-4.87)$ & $(-3.51)$ & $(-4.92)$ & $(-4.38)$ \\
$\theta_{1}$ & $-0.119^{* * *}$ & $0.0000314^{*}$ & -0.00034 & $-0.00407^{* * *}$ & $-0.188^{* * *}$ \\
& $(-3.41)$ & -1.92 & $(-0.11)$ & $(-3.86)$ & $(-4.70)$ \\
$\varphi_{2}^{\prime}$ & $1.518^{* * *}$ & $1.542^{* * *}$ & $1.511^{* * *}$ & $1.497^{* * *}$ & $1.498^{* * *}$ \\
& -7.58 & -7.68 & -7.54 & -7.46 & -7.46 \\
$\varphi_{1}^{\prime}$ & $-1.193^{*}$ & $-0.00117^{* * *}$ & $-0.0952^{* * *}$ & $-0.0454^{* * *}$ & $-1.818^{* * *}$ \\
& $(-1.68)$ & $(-5.05)$ & $(-3.00)$ & $(-4.11)$ & $(-3.50)$
\end{tabular}

Panel D. Mediating variable MCOST - Innovation Measured by RDIN

\begin{tabular}{llllll}
\hline \multirow{2}{*}{$\varphi_{1}$} & $-0.0568^{* * *}$ & -0.00000945 & $-0.00270^{* *}$ & $-0.00188^{* * * *}$ & $-0.0819^{* * *}$ \\
& $(-3.91)$ & $(-1.37)$ & $(-2.05)$ & $(-3.92)$ & $(-3.97)$ \\
$\theta_{1}$ & $-0.119^{* * *}$ & $0.0000314^{*}$ & -0.00034 & $-0.00407^{* * *}$ & $-0.188^{* * *}$ \\
& $(-3.41)$ & -1.92 & $(-0.11)$ & $(-3.86)$ & $(-4.70)$ \\
$\varphi_{2}^{\prime}$ & $0.254^{* * * *}$ & $0.254^{* * *}$ & $0.254^{* * *}$ & $0.253^{* * *}$ & $0.254^{* * *}$ \\
& -27.76 & -27.81 & -27.75 & -27.74 & -27.73 \\
$\varphi_{1}^{\prime}$ & -0.0211 & $-0.0000169^{* * *}$ & -0.000161 & -0.000305 & -0.00247 \\
& $(-1.62)$ & $(-2.89)$ & $(-0.14)$ & $(-0.80)$ & $(-0.15)$ \\
\hline
\end{tabular}

t statistics in parentheses, ${ }^{*} \mathrm{p}<0.1,{ }^{* *} \mathrm{p}<0.05,{ }^{* * *} \mathrm{p}<0.01$ 
The analysis process includes the following five steps (Wen and Ye, 2014): Step one is estimating equation (9). If $\varphi_{1}$ is statistically significant, it indicates that the stock liquidity affects the firm innovation; Step two is estimating equation (10) and (11). If the coefficient $\theta_{1}$ in equation (10) and coefficient $\varphi_{2}^{\prime}$ in equation (11) are both statistically significant, skip the step three and step four and go directly to the step five; If one or both of the coefficient $\theta_{1}$ and coefficient $\varphi_{2}^{\prime}$ are not statistically significant, continue the step three; Step three is testing the significance of $\theta_{1} \varphi_{2}^{\prime}$ by using the Bootstrap approach. If it is not significant, it indicates that the mediating effect is not significant. If it is significant, go to the next step; Step four is testing the significance of $\varphi_{1}^{\prime}$. If it is not significant, it indicates that there is only direct effect instead of mediating effect. If it is significant, continue to the next step; Step five is comparing $\theta_{1} \varphi_{2}^{\prime}$ and $\varphi_{1}^{\prime}$. If they have different sign, it indicates that there is masking effect. If they have the same sign, it suggests that the mediating variable may be an underlying mechanism through which stock liquidity enhances firm innovation.

\subsection{Financial Constraints}

Because of the limited and unstable supply of internal funds and the high-level asset specificity and information asymmetry of innovation activities, it is more likely to be affected by external financing constraints. High stock liquidity may positively affect firm innovation through the financing constraints mechanism, thus providing more external funds to innovation activities.

Considering that the higher the stock liquidity, the lower the financing cost, and firms more likely to increase equity financing which would lower the financial leverage (Lipson \& Mortal, 2009; Gu \& Chen,2009), I use the capital structure (asset-liability ratio) as the proxy of financial constraints to test the financial constraint mechanism.

Table 5 reports the results. Panel A reports the regression result of the mediating model which the dependent variable is LNRD and mediating variable is LEV. $\varphi_{1}$, the coefficients on LEV in equation (10), are negative and both economically and statistically significant. It suggests that stock liquidity negatively affects firms' financial constraints. $\theta_{1}$ in equation (10) and coefficient $\varphi_{2}^{\prime}$ in equation (11) are both statistically significant. $\varphi_{1}^{\prime}$ in equation (11) is statistically significant and $\theta_{1} \varphi_{2}^{\prime}$ and $\varphi_{1}^{\prime}$ have the same sign. It suggests that financial constraints may be an underlying mechanism through which stock liquidity enhances firm innovation. Panel B of table 5 reports the regression results with the dependent variable replaced by RDIN. The results are consistent with those in Panel A. My evidence suggests that high stock liquidity may enhance firm innovation through decreasing the firms's financial constraints.

\subsection{Agency Costs}

The agency problem exists in the innovation investment. The motivation of controlling more resources to gain more personal interests can impel executives to overinvest. The liquidity of the stock can affect the agency cost. On one hand, when stock liquidity is high, executives tend to reduce opportunism behaviour in order to reduce the adverse impact by the change of liquidity on their income. But on the other hand, higher liquidity reduces the costs of 'exit' of large shareholders and institutional investors (Bhide, 2004). On the contrary, when the stock liquidity is low, there is an exit barrier which may lead to "forced supervision".

Panel $\mathrm{C}$ of Table 5 reports the regression result of the mediating model which the dependent variable is LNRD and mediating variable is MCOST. $\varphi_{1}$, the coefficients on MCOST in equation (10), are positive and both economically and statistically significant, with only one exception that when I use LIU as stock liquidity measure it is negative. It suggests that stock liquidity negatively affects firms' agency cost. $\theta_{1}$ in equation (10) and coefficient $\varphi_{2}^{\prime}$ in equation (11) are both statistically significant. $\varphi_{1}^{\prime}$ in equation (11) is statistically significant and $\theta_{1} \varphi_{2}^{\prime}$ and $\varphi_{1}^{\prime}$ have the same sign. It suggests that agency costs may be an underlying mechanism through which stock liquidity enhances firm innovation. Panel D of table 5 reports the regression results with the dependent variable replaced by RDIN. The results are consistent with those in Panel C. My evidence suggests that high stock liquidity may enhance firm innovation through increasing the firm's agency costs. 


\section{Conclusion}

Based on existing literatures, through theoretical analysis and logical reasoning, I hypothesized that stock liquidity can affect firm innovation. By studying Chinese A shares of non-financial listed companies from 2006-2016, this paper analyses the influence of stock liquidity on firm innovation in the Chinese market empirically. The results of basic OLS regression analysis suggest that stock liquidity will enhance firm innovation. To alleviate the endogeneity problem, I then use the Heckman two-stage model. In addition, I analyse the hypothesized mechanisms through which stock liquidity may affects firm innovation. Overall, my study results show that (i) there is a significantly positive relationship between stock liquidity and firm innovation. (ii) stock liquidity can affect firm innovation through financing constraints mechanism. Higher stock liquidity reduces financing constraints and then enhance firm innovation. (iii) stock liquidity can affect firm innovation through agency costs mechanism. Higher stock liquidity increases agency cost and then enhance firm innovation.

My study results have several policy implications for government to encourage firm innovation. The change of financial market regulations and policies will affect the stock liquidity, and then stock liquidity can affect firm innovation. It suggests that the government should improve the capital market system, accelerate the construction of multi-level capital market system. Stock market regulators should strengthen the regulation of capital market and protect the interests of investors, in order to improve liquidity.

How to make full use of stock liquidity in enhancing firm innovation through alleviating the financing constraints rather than being through increasing agency costs need to be further studied. Besides, it should be pointed out that my study results are based on the listed firms that disclose the $R \& D$ expenditures data in China where there are quite unique characteristics of market operation. Therefore, the generality of the study findings should be cautious when making inference to non-listed firms in China or to samples in other countries with substantially different market conditions.

\section{References}

Amihud, Y. (2002). Illiquidity and stock returns: cross-section and time-series effects. Journal of Financial Markets, 5(1), 31-56. https://doi.org/10.1016/S1386-4181(01)00024-6

Amihud, Y., \& Mendelson, H. (1986). Asset pricing and the bid-ask spread. Journal of Financial Economics, 17(2), 223-249. https://doi.org/10.1016/0304-405X(86)90065-6

Bhide, A. (2004). The hidden costs of stock market liquidity. Journal of Financial Economics, 34(1), 31-51. https://doi.org/10.1016/0304-405X(93)90039-E

Chen, X. M., \& Wang, X. T. (2016). Government subsidies, financial constraints and corporate innovation. Science and Technology Management Research (in Chinese), 36(6), 11-18.

Chen, Y., Li, X. P., \& Bai, P. (2007). How market structure influence R\&D input: an empirical analysis on china's manufacture's panel data. Nankai Economic Studies (in Chinese), 1, 135-145.

Cheung, W. M., Chung, R., \& Fung, S. (2015). The effects of stock liquidity on firm value and corporate governance: Endogeneity and the REIT experiment. Journal of Corporate Finance, 35, 211-231. https://doi.org/10.1016/j.jcorpfin.2015.09.001

Dass, N., Nanda, V., K., \& Xiao, S. C. (2012). Do firms choose their stock liquidity? a study of innovative firms and their stock liquidity. SSRN Electronic Journal. https://doi.org/10.2139/ssrn.2131993

Fang, V. W., Tian, X., \& Tice, S. (2014). Does stock liquidity enhance or impede firm innovation. The Journal of Finance, 69(5), 2085-2125. https://doi.org/10.1111/jofi.12187

Feng, G. H., Liu, H., Feng, Z. Z., \& Wen, J. (2017). Does stock liquidity enhance technological innovation. Journal of Financial Research (in Chinese), 3, 192-206.

Gu, N. K., \& Chen, H. (2009). Stock liquidity and the determination of capital structure of enterprises: evidence from china's listed companies. Journal of Finance and Economics (in Chinese), 8, 37-48.

Holmstrom, B. (1989). Agency costs and innovation. Journal of Economic Behaviour \& Organization, 12(3), 305-327. https://doi.org/10.1016/0167-2681(89)90025-5

Lai, V. (2013). Stock market liquidity and innovation activity. SSRN Electronic Journal.

Lesmond, D. A., Ogden, J. P., \& Trzcinka, C. A. (1999). A new estimate of transaction costs. Review of Financial Studies, 12(5), 1113-1141. https://doi.org/10.1093/rfs/12.5.1113

Lipson, M. L., \& Mortal, S. (2007). Capital structure decisions and equity market liquidity. SSRN Electronic 
Journal.

Liu, S. Q., Lin, Z. J. Shun, F. C., \& Chen, H. W. (2015). The impact of financing constraints and agency costs on the corporaterd \& investment in China. Accounting Research (in Chinese), 11, 62-68.

Liu, W. (2006). A liquidity-augmented capital asset pricing model. Journal of Financial Economics, 82(3), 631-671. https://doi.org/10.1016/j.jfineco.2005.10.001

Lu, T., \& Dang, Y. (2014). Corporate governance and innovation: differences among industry categories. Economic Research Journal (in Chinese), 6, 115-128.

Lu, X., Zheng, Y. F., \& Li, J. M. (2013). Research on the impact of financing constraints on corporate R\&D Investment -- evidence from the Hi-tech listed companies in China. Accounting Research (in Chinese), 5, 51-58.

Luo, Z. Y., Li, Y. J., \& Chang, J. (2014). Study on the conduction effect of private enterprises' stockholder's rights structure upon R\&D investment behaviour. Soft Science (in Chinese), 3, 167-176.

Lv, X. J. (2013). Agency conflicts, incentive compatibility and technology innovation of Chinese listed companies: empirical analysis based on two-tier stochastic frontier model. Modern Finance and Economics-Journal of Tianjin University of Finance and Economics (in Chinese), 11, 118-128.

Lv, X. J. (2014). Effect of agency conflicts on the innovation of listed companies in China. Science Research Management (in Chinese), 11, 60-67.

Pastor, L., \& Stambaugh, R. F. (2002). Liquidity risk and expected stock returns. Social Science Electronic Publishing, 111(3), 642-685.

Wen, Z. L. \& Ye, B. J. (2014). Analyses of mediating effects: the development of methods and models. Advances in Psychological Science (in Chinese), 22(5), 731-745. https://doi.org/10.3724/SP.J.1042.2014.00731

Xiao, L. P. (2016). How corporate governance influences firm's R\&D investment? -- Empirical evidence from strategic emerging industries in China. Industrial Economics Research (in Chinese), 1, 60-70.

Xiong, J. C., \& Su, D. W. (2014). Stock liquidity and capital allocation efficiency. Accounting Research (in Chinese), 11, 54-60.

Xiong, J. C., \& Su, D. W. (2016). Ownership structure, stock liquidity and agency costs: a stochastic frontier approach. Nankai Business Review (in Chinese), 1, 84-96.

Zhang, J., Lu, Z., Zheng, W. P., \& Chen, Z. Y. (2012). Financing constraints, financing channels and firm R\&D investment. The Journal of World Economy (in Chinese), 10, 66-90.

\section{Copyrights}

Copyright for this article is retained by the author(s), with first publication rights granted to the journal.

This is an open-access article distributed under the terms and conditions of the Creative Commons Attribution license (http://creativecommons.org/licenses/by/4.0/). 